\title{
Differential expression of DUSP2 in left- and right-sided colon cancer is associated with poor prognosis in colorectal cancer
}

\author{
WENJIE DONG ${ }^{1 *}, \mathrm{NA} \mathrm{LI}^{1 *}$, XIUFENG PEI ${ }^{2}$ and XINAI WU ${ }^{1}$ \\ ${ }^{1}$ Department of Internal Medicine-Oncology, The First Affiliated Hospital, Zhengzhou University, Zhengzhou, Henan 450052; \\ ${ }^{2}$ Department of Internal Medical Oncology, Tumor Hospital of Baotou, Baotou, Inner Mongolia 014030, P.R. China
}

Received May 16, 2017; Accepted December 8, 2017

DOI: $10.3892 / \mathrm{ol} .2018 .7881$

\begin{abstract}
Dual-specificity phosphatase-2 (DUSP2), a negative regulator of extracellular-regulated kinase activity, has been identified as an important kinase with emerging roles in cancer. However, the clinical significance of DUSP2 in colorectal cancer (CRC) remains to be fully elucidated. In the present study, the expression of DUSP2 was investigated using immunohistochemistry in 96 patients with CRC. Cell viability was estimated using a cell counting kit-8 assay, and cell apoptosis by flow cytometry. The relationship between DUSP2 expression and patient characteristics, including overall survival, were studied retrospectively in these patients. It was found that DUSP2 was differentially expressed between left-sided colon carcinoma (LSCC) and right-sided colon carcinoma (RSCC). It was also found that decreased expression of DUSP2 was correlated with significantly shorter overall survival $(\mathrm{P}=0.001)$ and short distant-metastasis-free survival $(\mathrm{P}=0.002)$. In univariate comparisons, the decreased expression of DUSP2 was found to be an independent risk factor for poor survival rate (HR 3.55, CI 1.092-9.896; $\mathrm{P}=0.002$ ). It was also found that the enforced overexpression of DUSP2 sensitized CRC cells to cetuximab. In conclusion, the findings demonstrated that DUSP2 was differentially expressed between RSCC and LSCC, and that the overexpression of DUSP2 increased the inhibitory effect of cetuximab in CRC, suggesting that DUSP2 may be a novel biomarker and therapeutic target in CRC therapy.
\end{abstract}

\section{Introduction}

Dual specificity phosphatase 2 (DUSP2) is a member of the dual-specificity phosphatases, which specifically inactivates

Correspondence to: Dr Xinai Wu, Department of Internal Medicine-Oncology, The First Affiliated Hospital, Zhengzhou University, 1 East Jianshe Road, Zhengzhou, Henan 450052, P.R. China

E-mail: wuxinaizdyfy@163.com

${ }^{*}$ Contributed equally

Key words: dual-specificity phosphatase-2, colorectal cancer, prognosis, cetuximab mitogen-activated protein kinase (MAPK) signaling by the direct dephosphorylation of phosphothreonine and phosphotyrosine residues $(1,2)$. DUSP2 is expressed at high levels in immune cells, particularly in patients with inflammatory arthritis, including rheumatoid arthritis, and DUSP2-knockout mice present with a significant reduction in inflammatory responses (3). DUSP2 is crucial in regulating the tumor-relevant MAPK pathways. These pathways drive proliferation, differentiation via the regulation of MAP kinases, extracellular-signal regulated kinase (ERK)1/2, p38 and c-Jun N-terminal kinase (JNK), and apoptosis (4). For example, DUSP2 is a transcription target of p53 and E2F1 in signaling apoptosis and growth suppression (5). It is reported that DUSP2 is epigenetically silenced by promoter methylation in several cancer cell lines, including skin and lung cancer (6). It is also reported that DUSP2 is suppressed by hypoxia and the loss of function of DUSP2 not only leads to the prolonged activation of ERK and tumorigenesis, but also contributes to drug resistance $(7,8)$. For example, hypoxia induces lapatinib resistance in Erb-B2 receptor tyrosine kinase 2-positive breast cancer cells via the regulation of DUSP2 (9).

Several studies have reported that DUSP2 may act as a tumor suppressor $(5,10,11)$, whereas others have reported that it may be involved in promoting cancer progression (12). For example, a high mRNA expression level of DUSP2 predicted significantly poorer overall survival rates, compared with low expression in serous ovarian carcinoma (12). Therefore, the role of DUSP2 appears to vary with the type of malignancy. However, whether DUSP2 acts as a tumor promoter or tumor suppressor is controversial.

The expression of DUSP2 is downregulated in several types of human cancer, and the loss of DUSP2 promotes cancer progression. However, the clinical value of DUSP2 in patients with colorectal cancer (CRC) remains to be elucidated. The present study, using a retrospective CRC patient cohort, aimed to examine the biological function and clinical significance of DUSP2 in CRC.

\section{Materials and methods}

Patients and tissue microarray. The samples examined in the present study consisted of 96 patients with CRC, which were obtained from the tissue specimen bank of Shanghai Biological Technology Co., Ltd. (Shanghai, China). The patient 
surgery was performed between July 2006 and May 2007, and follow-up was continued until August 2014. The clinicopathological classification was determined according to the tumor-node-metastasis (TNM) classification of malignant tumors. All the patients were pathologically diagnosed with colorectal cancer without any pre-surgical treatment. There were 51 men and 45 women, with a median age of 55 years. Each study specimen of cancer tissue was provided with adjacent-carcinoma tissue, which was sited at a distance of $1.5 \mathrm{~cm}$ from the cancer tissue.

Immunochemical (IHC) staining. The tissue samples were processed as formalin-fixed, paraffin-embedded tissue specimens according to standard institutional procedures. Sections (4- $\mu \mathrm{m}$ thick) were cut from the paraffin-embedded tissue specimens and used for the IHC. Sections were heat-immobilized at $60^{\circ} \mathrm{C}$ for $30 \mathrm{~min}$ and deparaffinized in xylene and rehydrated through a series of graded ethanol solutions $(100,95,90,80$ and $70 \%)$ at room temperature for $10 \mathrm{~min}$. Antigen retrieval was performed in a pressure cooker at $95^{\circ} \mathrm{C}$ for 2 min using $0.01 \mathrm{M}$ citrate buffer ( $\mathrm{pH}$ 6.0). The samples were analyzed under x400 magnification using a BX51 light microscope (Olympus Corporation, Tokyo, Japan). The IHC results were reviewed by two expert pathologists. The specimens were then divided into four grades, according to the degree of positivity as follows: Grade 0 , grade 1 (1-25\% positive), grade 2 (26-50\% positive) and grade 3 (51-100\% positive). For the statistical analyses, grades 0 and 1 were defined as negative, and grades 2 and 3 were defined as positive.

The cancer genome atlas (TCGA) data analysis. The data files used to analyze DUSP2 expression were initially downloaded from the TCGA (http://cancergenome.nih.gov/) data portal website, by using the data matrix link to access RNASeq data and by using the UNC (IlluminaHiSeq_RNAseqV2) data platform. DUSP2 mRNA expression data from 461 CRC patients were obtained from the TCGA.

Immunofluorescence. For the immunofluorescence analyses, the cells were cultured in 6-cm dishes. The cells were mounted with cytospin on polylysine-coated glass slides and fixed with 4\% paraformaldehyde for $15 \mathrm{~min}$, followed by the addition of $100 \%$ ice-cold acetone for $10 \mathrm{~min}$ at $4{ }^{\circ} \mathrm{C}$. To detect the protein expression of DUSP2, immunofluorescence analysis was performed with DUSP2 antibody (dilution, 1:100; cat. no. LS-B14289; LifeSpan BioScienes, Inc.), incubated for $24 \mathrm{~h}$ at $4^{\circ} \mathrm{C}$, followed by incubation with anti-IgG-PE (Santa Cruz Biotechnology, Inc., Dallas, TX, USA) at $37^{\circ} \mathrm{C}$ for $30 \mathrm{~min}$ in the dark and mounting with DAPI mounting medium. The samples were investigated by laser scanning confocal microscopy (OLS4100; Olympus Corporation, Tokyo, Japan).

Cell lines. The colon cancer cell line, SW48, was obtained from the Shanghai Cell Bank Chinese Academy of Sciences (Shanghai, China). SW48 cells were cultured in RPMI-1640 medium (Gibco; Thermo Fisher Scientific, Inc., Waltham, MA, USA) supplemented with $10 \%$ fetal bovine serum (Hyclone; GE Healthcare Life Sciences, Logan, UT, USA), 100 U/ml penicillin and $100 \mathrm{U} / \mathrm{ml}$ streptomycin (Invitrogen; Thermo Fisher Scientific, Inc.), at $37^{\circ} \mathrm{C}, 5 \% \mathrm{CO}_{2}$ and $95 \%$ humidity. The human colorectal carcinoma cell lines, HCT116 and HCT15, were purchased from the Shanghai Institutes for Biological Sciences, Chinese Academy of Sciences (Shanghai, China), and were cultured in RPMI-1640 medium (Gibco; Thermo Fisher Scientific, Inc.) supplemented with $10 \%$ fetal bovine serum (Hyclone; GE Healthcare Life Sciences), $100 \mathrm{U} / \mathrm{ml}$ of penicillin and $100 \mathrm{U} / \mathrm{ml}$ of streptomycin (Invitrogen; Thermo Fisher Scientific, Inc.), at $37^{\circ} \mathrm{C}, 5 \% \mathrm{CO}_{2}$ and $95 \%$ humidity.

SiRNA transfection. SW48 cells, cultured in RPMI-1640 medium (Gibco; Thermo Fisher Scientific, Inc.) supplemented with $10 \%$ fetal bovine serum (Hyclone; GE Healthcare Life Sciences), were transfected with siRNAs targeting DUSP2 or with control siRNA using Lipofectamine ${ }^{\circledR} 2000$ (Invitrogen; Thermo Fisher Scientific, Inc.). Cells were transfected with siRNA at a concentration of $50 \mathrm{nM}$. The transfection was performed at $37^{\circ} \mathrm{C}$ in a humidified incubator with $5 \% \mathrm{CO}_{2}$. The siRNAs were designed and synthesized by Shanghai GenePharma Co., Ltd. (Shanghai, China). The sequences of the siRNAs used in the present study were as follows: siRNA-DUSP2 sense, 5-GCAUCACAGCCGUCCUCAATT-3 and anti-sense, 5-UUGAGGACGGCUGUGAUGCTT-3, and NC-siRNA sense, 5-GCAACACCGCUGUCUCCAATT-3 and anti-sense, 5-UUGGAGACAGCGGUGUUGCTT-3. When SW48 cells reached $80 \%$ confluence 6-well plates, transfection was conducted by mixing $5 \mu \mathrm{l}$ siRNA with $5 \mu \mathrm{l}$ Lipofectamine ${ }^{\circledR} 2000$ in a final volume of $2,000 \mu 1$ medium. Cell morphology and transfection efficiency were evaluated after $6 \mathrm{~h}$ transfection. Transfections were performed in triplicate and each experiment was repeated $\geq 3$ times.

Reverse transcription-quantitative (RT-qPCR). Total RNA was isolated using TRIzol reagent (Thermo Fisher Scientific, Inc.), and reverse transcription of total RNA was carried out using MMLV-RT, SPCL (Invitrogen; Thermo Fisher Scientific, Inc.). Quantitative PCR was performed by using SYBR Green I (Thermo Fisher Scientific, Inc.). The reaction mixture consisted of $2.5 \mu \mathrm{l}$ 10X PCR buffer, $2.0 \mu \mathrm{l}$ of $2.5 \mathrm{mM}$ each dNTP, $2.0 \mu \mathrm{l}$ $25 \mathrm{mM} \mathrm{MgCl} 2,0.5 \mu 110 \mathrm{pmol} / \mu 1$ each primer, $0.2 \mu 15 \mathrm{U} / \mu 1$ Taq polymerase, $0.5 \mu 1 \mathrm{cDNA}$ template and distilled water for a total volume of $25 \mu \mathrm{l}$. The primer sequences used were as follows, human GAPDH, forward 5'-CCACCCATGGCAAAT TCCATGGCA-3' and reverse 5'-TCTAGACGGCAGGTC AGGTCCAC-3'; DUSP2, forward 5'-TTTGAGGGCCTTTTC CGCTACAAGAG-3' and reverse 5'-GCCTCCGCTGTTCTT CACCCAGTC-3' (6). The thermocycling conditions were as follows: $94^{\circ} \mathrm{C}$ for $5 \mathrm{~min} ; 35$ cycles of $94^{\circ} \mathrm{C}$ for $30 \mathrm{sec}, 56^{\circ} \mathrm{C}$ (GAPDH) $/ 65^{\circ} \mathrm{C}$ (DUSP2) for $30 \mathrm{sec}$, and $72^{\circ} \mathrm{C}$ for $60 \mathrm{sec}$, and a final extension at $72^{\circ} \mathrm{C}$ for $10 \mathrm{~min}$. Triplicate tests were performed for each sample, and all reactions were repeated 3 times independently to ensure reproducibility of results. The data were then viewed and analyzed using the Rotor-Gene Real-Time Analysis Software (Corbett Rotor-Gene 6000; Qiagen, Doncaster, Australia). For each sample, amplification plot and corresponding dissociation curves were examined. To obtain standardized quantitative results, external controls were constructed consisting of cDNA plasmid standards (13).

hEGF treatment. HCT116 and HCT15 cells (3x10\%/well) were seeded in 6-well plates. After $24 \mathrm{~h}$, the cells were treated 
with $100 \mathrm{ng} / \mathrm{ml} \mathrm{hEGF}$ for another $24 \mathrm{~h}$. Then the protein was extracted and subjected to western blot analysis.

Western blot analysis. The cells were harvested and centrifuged at $110 \mathrm{x} \mathrm{g}$, for $5 \mathrm{~min}$ at room temperature. The supernatant was removed, and the cell pellet was washed twice with PBS (0.01 M; pH 7.2-7.3). Each tube of cells was added into $100 \mu \mathrm{l}$ radioimmunoprecipitation assay lysis buffer with phenylmethane sulfonyl fluoride (Sigma-Aldrich; Merck KGaA, Darmstadt, Germany), and protein concentrations were using a bicinchoninic acid assay. The samples $(20 \mu \mathrm{g})$ of the cell lysate were subjected to $10 \%$ SDS-PAGE gel electrophoresis, following which the resolved proteins were transferred onto nitrocellulose membranes (GE Healthcare Life Sciences, Chalfont, UK). The membranes were then blocked with 5\% non-fat milk and $0.1 \%$ Tween 20 in Tris-buffered saline, and probed with anti-DUSP2 antibody (dilution, 1:500; cat. no. LS-B14289; LifeSpan BioScienes, Inc.), and a secondary antibody for $60 \mathrm{~min}$ at room temperature [goat anti-rabbit monoclonal antibody (dilution; 1:8,000; cat. no. SA00001-1; ProteinTech Group, Inc., Chicago, IL, USA)], following which the blots were visualized using enhanced chemiluminescence (GE Healthcare Life Sciences).

Evaluation of apoptosis. Apoptosis was detected via flow cytometric analysis of Annexin V staining. The Annexin V-FITC/PI assay was performed as previously reported (14). Briefly, the adherent cells were harvested and suspended in the Annexin-binding buffer $\left(1 \times 10^{6}\right.$ cells $\left./ \mathrm{ml}\right)$. The cells were then incubated with Annexin V-FITC and PI for $15 \mathrm{~min}$ at room temperature in the dark, and immediately analyzed via flow-cytometry. The data are presented as bi-parametric dot plots showing Annexin V-FITC green fluorescence, vs. PI red fluorescence.

Cell viability assay. The cells were seeded at a density of 3,000 cells/well in 96-well plates. When the cells had completely adhered to the well, the culture medium was replaced with medium containing $10 \%$ FBS and a certain concentration of cetuximab $(0,1,2.5,5$ or $10 \mu \mathrm{g} / \mathrm{ml})$, and cultivated at $37^{\circ} \mathrm{C}, 5 \% \mathrm{CO}_{2}$ for $48 \mathrm{~h}$. After $2 \mathrm{~h}$, cell viability was measured using a Cell Counting Kit-8 (CCK-8), according to the manufacturer's protocol. Briefly, a mixture of $10 \mu \mathrm{l}$ CCK-8 (Dojindo Molecular Technologies, Inc.) and $190 \mu \mathrm{l}$ of RPMI-1640 with $10 \%$ FBS was added to each well. An MRX II microplate reader was used to measure the optical density at $450 \mathrm{~nm}$. A background reading of the media was subtracted from each well to standardize the results. Optical density (OD) was utilized as an indicator of cell survival.

Statistical analysis. To investigate the associations between relapse-free survival rate and various clinicopathological factors, survival analysis was performed using the Kaplan-Meier method, and the differences were evaluated using the log-rank test. A multivariate survival analysis was performed using Cox's proportional-hazard model. Hazard ratios and $95 \%$ confidence intervals were used to measure associations. SPSS 13 software (SPSS, Inc., Chicago, IL, USA) was used for all statistical analyses. $\mathrm{P}<0.05$ was considered to indicate a statistically significant difference.
Table I. Association between the clinicopathological features of patients with colorectal cancer and the expression levels of DUSP2.

\begin{tabular}{|c|c|c|c|}
\hline \multirow[b]{2}{*}{ Variable } & \multicolumn{2}{|c|}{ Expression of DUSP2 } & \multirow[b]{2}{*}{ P-value } \\
\hline & $\begin{array}{c}\text { Positive } \\
(n=69)\end{array}$ & $\begin{array}{c}\text { Negative } \\
(n=27)\end{array}$ & \\
\hline \multicolumn{4}{|l|}{ Age (years) } \\
\hline$\leq 60$ & 32 & 16 & 0.785 \\
\hline$>60$ & 37 & 11 & \\
\hline \multicolumn{4}{|l|}{ Gender } \\
\hline Male & 33 & 18 & 0.665 \\
\hline Female & 36 & 9 & \\
\hline \multicolumn{4}{|c|}{ Primary tumor $(\mathrm{T})$} \\
\hline $\mathrm{T} 1 / \mathrm{T} 2$ & 31 & 12 & 0.572 \\
\hline T3/T4 & 38 & 15 & \\
\hline \multicolumn{4}{|c|}{ Nodal status (N) } \\
\hline No & 30 & 12 & 0.563 \\
\hline $\mathrm{N} 1 / \mathrm{N} 2 / \mathrm{N} 3$ & 39 & 15 & \\
\hline \multicolumn{4}{|l|}{ Stage } \\
\hline I-II & 37 & 15 & 0.671 \\
\hline III-IV & 32 & 12 & \\
\hline \multicolumn{4}{|c|}{ Primary tumor site } \\
\hline RSCC & 26 & 20 & 0.008 \\
\hline LSCC & 43 & 7 & \\
\hline
\end{tabular}

DUSP2, dual specificity phosphatase 2 ; LSCC, left-sided colon carcinoma; RSCC, right-sided colon carcinoma.

\section{Results}

DUSP2 is differentially expressed between left-sided colon carcinoma (LSCC) and right-sided colon carcinoma (RSCC). To determine the expression of DUSP2 in CRC, the present study analyzed a tissue microarray containing primary CRC and paired adjacent normal tissues using IHC analysis. Several antibodies used for IHC are notoriously prone to false positive signals, and antibodies, which are capable of resolving a single positive band in western blots, can be found to stain non-specific targets in cells and tissue sections by IHC. The solution to this problem is to show that DUSP2 small interfering RNA attenuates or eliminates the DUSP2 signal (15). The silencing of DUSP2 was confirmed via western blot analysis in the SW48 cell line (Fig. 1A). The analysis was performed with DUSP2 antibody in SW48 control or DUSP2-silenced cells and mounted with DAPI mounting medium. The results indicated that the signals detected were those of DUSP2 (Fig. 1B). Overall, using a score to semi-quantify immunoreactivity, it was found that the majority of the CRC samples were graded as positive $(69 / 96,71.9 \%)$ and $34.27 \%$ were graded as negative, whereas fewer normal tissues were graded as positive (52/96, 54.2\%; $\mathrm{P}=0.016$; Fig. 1C). This was in contrast to previous findings that DUSP2 was reduced in certain types of cancer (6). The expression of DUSP2 in human CRC 


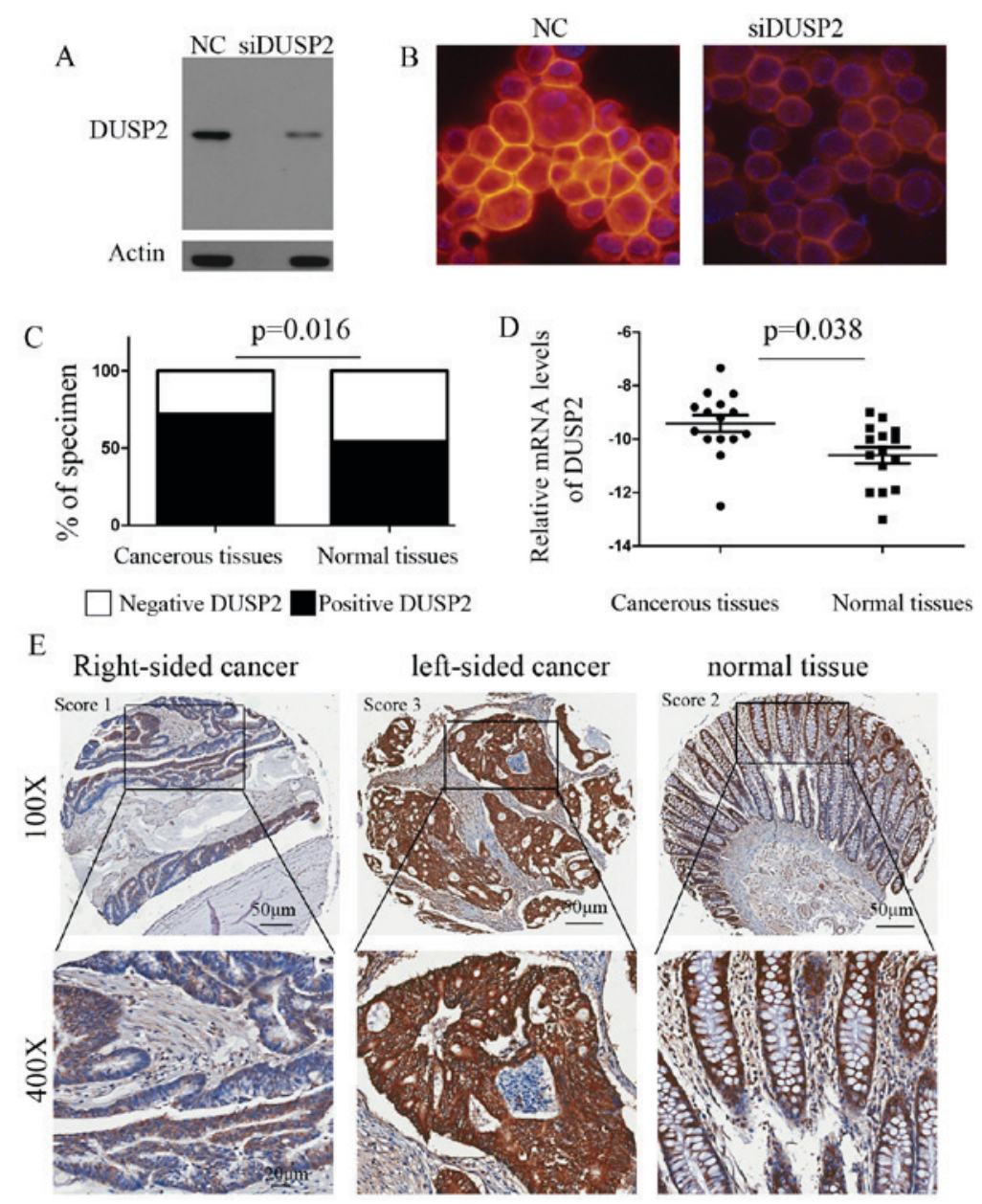

Figure 1. Immunohistochemical staining for DUSP2 in cancerous and normal tissues (A) Western blot analysis showing the efficiency of DUSP2 knockdown in SW48 cells. (B) Immunofluorescence analysis was performed with DUSP2 antibody in SW48 control or DUSP2-silenced cells and mounted with DAPI mounting medium (magnification, x1,000). (C) Protein expression levels of DUSP2 in clinical samples were determined using an IHC assay in patients with CRC. Quantification of the expression of DUSP2 according to IHC scores in 96 paired normal and tumor tissues, respectively. (D) Reverse transcription-quantitative polymerase chain reaction analysis of mRNA expression levels of DUSP2 in 15 paired normal and tumor tissues confirmed it was upregulated in tumor tissues. (E) Scores indicated levels of DUSP2 in representative tumor tissues. The scores were calculated by the intensity and percentage of stained cells. DUSP2, dual specificity phosphatase 2; CRC, colorectal cancer; NC, negative control; siDUSP2, small interfering RNA targeting DUSP2.

specimens was also analyzed using data of $461 \mathrm{CRC}$ patients from The Cancer Genome Atlas (TCGA; http://cancergenome .nih.gov). Amongst these, matched tumor and non-tumor specimens were available for 50 patients with CRC. To further validate the results, the total expression of DUSP2 was determined in 15 paired normal and tumor tissues via RT-qPCR analysis, which confirmed that DUSP2 was upregulated in tumor tissues (Fig. 1D; P=0.038). The correlations between the decreased expression of DUSP2 and the clinicopathological features were also examined. The expression of DUSP2 was not associated with age, gender, advanced TNM stage, nodal metastasis or depth of tumor invasion (Table I). The decreased expression of DUSP2 was significantly associated with primary tumor site $(\mathrm{P}=0.008)$. Compared with RSCC, the expression of DUSP2 was upregulated in LSCC. Representative images of the protein expression of DUSP2 in CRC and normal tissues are shown in Fig. 1E. Analysis of the relative gene expression of DUSP2 in all 50 patients with CRC from the TCGA gene expression datasets showed a significant increase in the mRNA expression of DUSP2 in tumor specimens, compared with that in non-tumor colorectal tissues (Fig. 2A and B).
Decreased expression of DUSP2 is correlated with poor prognosis in patients with CRC. The log-rank test and Kaplan-Meier survival curve were used to determine whether decreased expression of DUSP2 was correlated with overall survival rates and distant-metastasis-free survival (DMeFS). The results demonstrated that patients with CRC and a decreased expression of DUSP2 had a poorer overall survival rate, compared with patients with positive expression of DUSP2 (Fig. 3A). It was also found that a decreased expression of DUSP2 was correlated with significantly shorter DMeFS (Fig. 3B). To further evaluate the prognostic value of the expression of DUSP2, univariate Cox regression analysis was performed. The univariate Cox regression analysis indicated that the decreased expression of DUSP2 was an independent prognostic biomarker for CRC in patients (HR 3.55, CI 1.092-9.896; $\mathrm{P}=0.002$; Table II). To further confirm that the signals detected were those of DUSP2, the expression of DUSP2 was detected in tumor tissues using western blot analysis. The results revealed that the signal of DUSP2 in the IHC was consistent with the signal of DUSP2 obtained from the western blot analysis (Fig. 3C). 

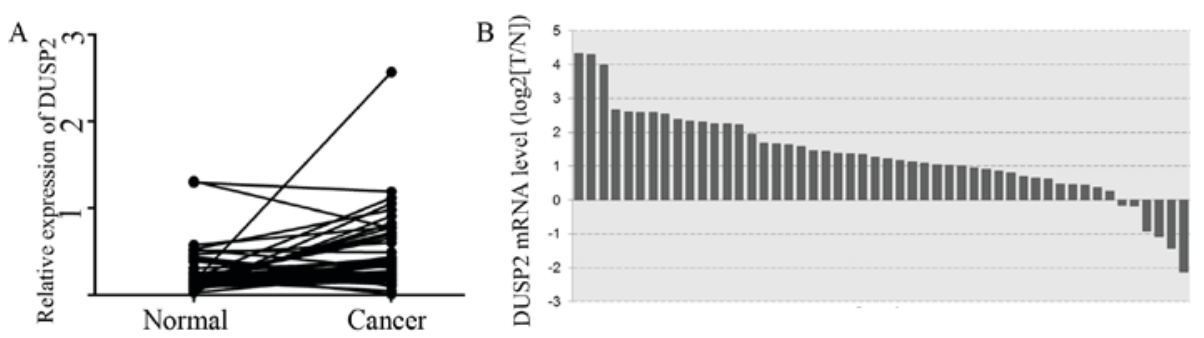

Figure 2. mRNA levels of DUSP2 in 50-paired tumor and non-tumor margin tissue specimens of patients with CRC obtained from The Cancer Genome Atlas gene expression profiling datasets are shown. mRNA levels of DUSP2 are expressed (A) individually in tumor, vs. non-tumor for each patient and as a (B) tumor/non-tumor ratio in each CRC patient. DUSP2, dual specificity phosphatase 2; CRC, colorectal cancer; T, tumor; N, normal.
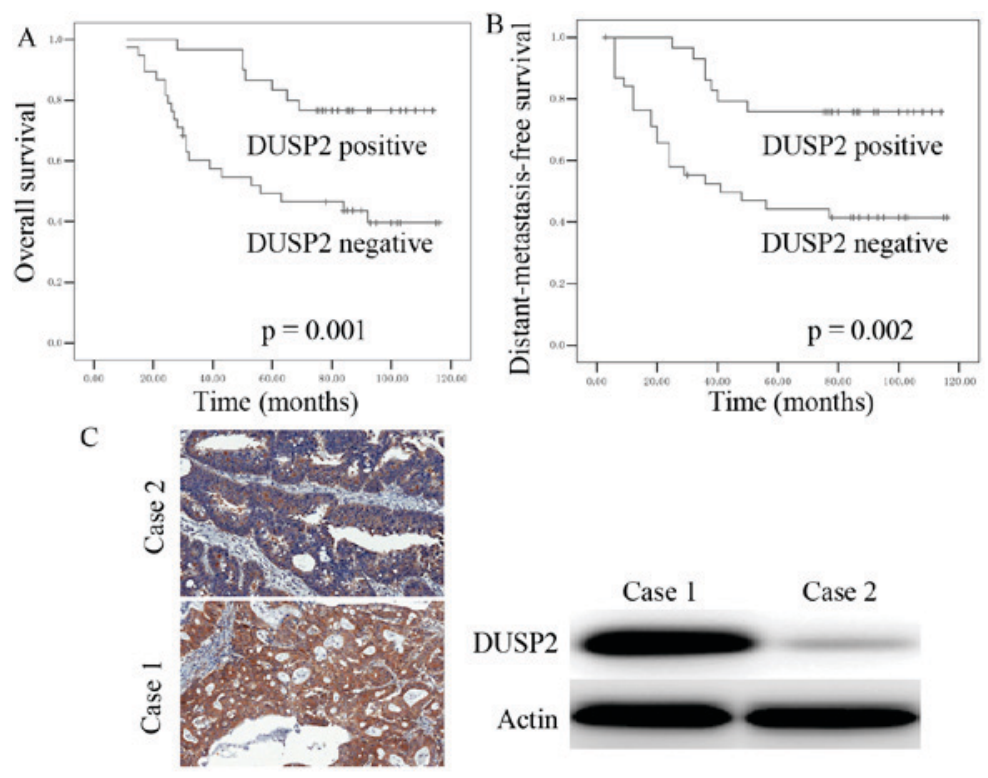

Figure 3. Kaplan-Meier survival analysis. Data revealed that a high expression of DUSP2 predicts (A) poorer overall survival and (B) distant-metastasis-free survival, respectively. (C) Representative images of DUSP2 immunohistochemistry (magnification, $\mathrm{x} 400$ ) and western blot analysis in human primary colorectal cancer specimens. DUSP2, dual specificity phosphatase 2 .

Table II. Univariate analysis of the expression of DUSP2 and overall survival in patients with colorectal cancer.

\begin{tabular}{|c|c|c|c|}
\hline \multirow[b]{2}{*}{ Variable } & \multicolumn{2}{|c|}{ Univariate analysis } & \multirow[b]{2}{*}{ P-value } \\
\hline & Hazard ratio & $95 \%$ CI & \\
\hline $\begin{array}{l}\text { Age (years) } \\
\geq 60, \text { vs. }<60\end{array}$ & 1.251 & $0.602-2.602$ & 0.548 \\
\hline Gender & & & \\
\hline Male, vs. female & 1.012 & $0.984-1.040$ & 0.405 \\
\hline $\begin{array}{r}\text { Primary tumor }(\mathrm{T}) \\
\mathrm{T} 1 / \mathrm{T} 2, \mathrm{vs} . \mathrm{T} 3 / \mathrm{T} 4\end{array}$ & 1.067 & $0.172-8.028$ & 0.545 \\
\hline $\begin{array}{l}\text { Nodal status }(\mathrm{N}) \\
\text { N0, vs. N1/N2/N3 }\end{array}$ & 2.071 & $0.473-6.404$ & 0.126 \\
\hline $\begin{array}{l}\text { Stage } \\
\text { I-II, vs. III-IV }\end{array}$ & 2.136 & $0.322-3.453$ & 0.235 \\
\hline $\begin{array}{l}\text { DUSP2 } \\
\text { Positive, vs. negative }\end{array}$ & 3.55 & $1.092-9.896$ & 0.002 \\
\hline
\end{tabular}

DUSP2, dual specificity phosphatase 2 .
Upregulation of DUSP2 is a consequence rather than a cause of tumor progression. DUSP2 specifically inactivates MAPK signaling by the direct dephosphorylation of phosphothreonine and phosphotyrosine residues of ERK and AKT. It is also reported that loss of function of DUSP2 leads to prolonged ERK activation. The present study hypothesized that the upregulation of DUSP2 may be a consequence rather than a cause of tumor progression in CRC cells. To address this question, the expression levels of DUSP2 and phosphorylated (p)ERK were analyzed using IHC to determine whether the expression of DUSP2 was positively correlated with pERK in CRC tissues. The results showed no significant correlation between DUSP2 and pERK (data not shown). It is well known that epidermal growth factor (EGF) stimulation causes a significant increase in pERK, and this effect is observed even following EGF withdrawal (16). In the present study, when the HCT15 and HCT116 cells were treated with $100 \mathrm{ng} / \mathrm{ml} \mathrm{hEGF}$ for $24 \mathrm{~h}$, it was found that the expression of DUSP2 was upregulated in the HCT15 cells. However, the treatment of HCT116 cells under the same conditions had no effect on the expression of DUSP2 (Fig. 4A). It has been reported that DUSP2 is epigenetically silenced in several cancer cells (6). The present study analyzed the promoter methylation status of DUSP2 in HCT15 and HCT116 


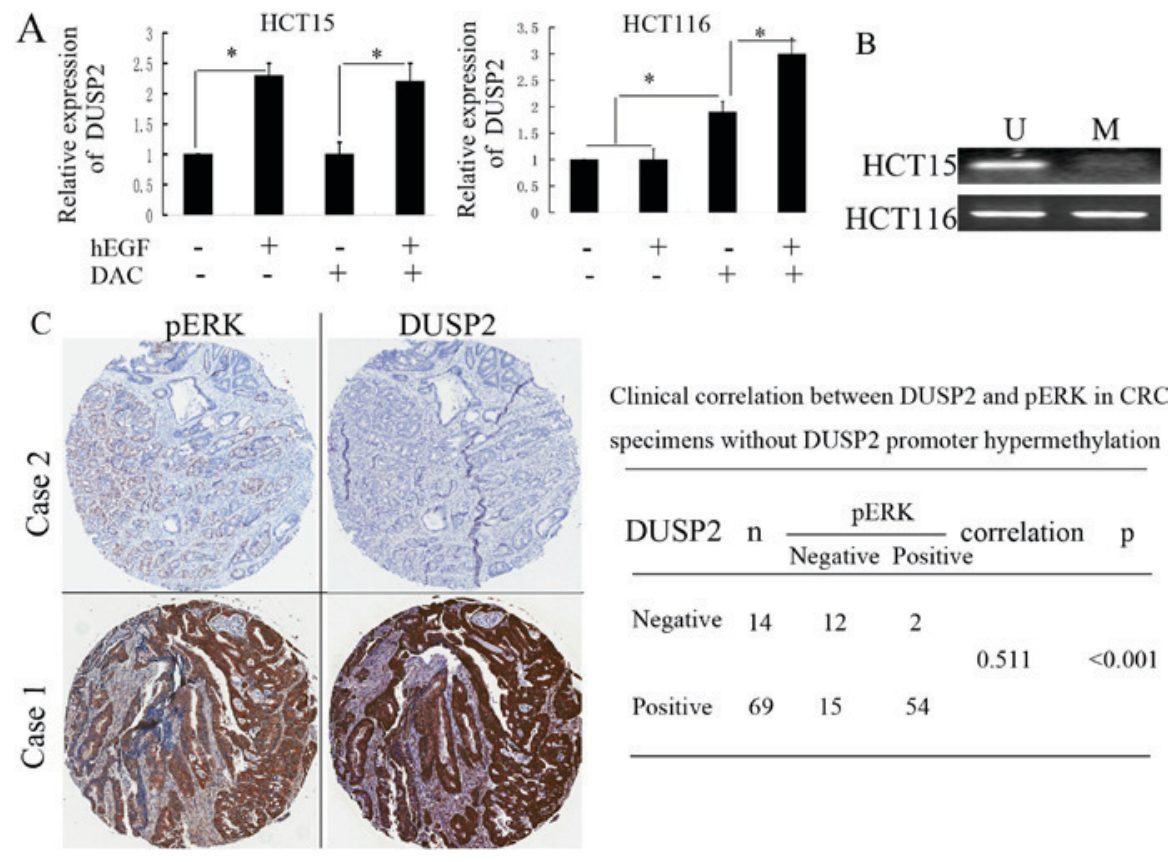

Figure 4. Upregulation of DUSP2 is a consequence rather than a cause of tumor progression. (A) Treatment of HCT15 and HCT116 cells with $100 \mathrm{ng} / \mathrm{ml} \mathrm{hEGF}$ for $24 \mathrm{~h}$. The expression of DUSP2 was upregulated in HCT15 but not HCT116 cells. Combined treatment with hEGF and DAC caused a synergistic induction of DUSP2 in HCT116 cells. (B) Promoter methylation status of DUSP2 in HCT15 and HCT116 cells was detected by methylation-specific polymerase chain reaction analysis. (C) A panel of 83 human CRC specimens was immunostained with DUSP2 and pERK, respectively. Representative DUSP2 and pERK immunohistochemical images of human primary CRC (magnification, $x 100$ ). The correlation between DUSP2 and pERK was analyzed using Pearson's $\chi^{2}$ test using the SPSS 13.0 statistical software package. A significant positive correlation between DUSP2 and pERK was found in CRC specimens without DUSP2 promoter hypermethylation. " $\mathrm{P}<0.05$. CRC, colorectal cancer; DUSP2, dual specificity phosphatase 2 ; pERK, phosphorylated extracellular signal-regulated kinase; EGF, epidermal growth factor; $\mathrm{U}$, unmethylated; $\mathrm{M}$, methylated.

cells via methylation-specific PCR (MSP). In accordance with the above-mentioned result, the hypermethylation of DUSP2 was observed in HCT116 cells, but not HCT15 cells (Fig. 4B). The treatment of HCT116 cells with EGF in combination with DAC, a methyltransferase inhibitor, markedly upregulated the expression of DUSP2 and exhibited a synergetic effect. The DUSP2 methylation status in CRC tissues was also detected by MSP. It was found that hypermethylation of DUSP2 was observed in 13 of 96 patients with CRC. The primary tumors of these 13 patients originated in the RSCC. The expression of DUSP2 in the cancer tissues from these 13 patients was negative. There was a significant correlation between promoter methylation and loss of DUSP2 in the RSCC $(\mathrm{P}<0.001)$. When the expression levels of DUSP2 and pERK were determined in CRC tissues without hypermethylation of DUSP2, it was found that the expression of DUSP2 was positively correlated with that of pERK in the CRC tissues (Fig. 4C). These results suggested that the hypermethylation of DUSP2 may inhibit the upregulation of DUSP2 induced by the increase of pERK. Although it is overexpressed in partial CRC tissues, the results suggested that DUSP2 functions as a tumor suppressor.

Enforced expression of DUSP2 sensitizes CRC cells to cetuximab treatment. It is also reported that the loss of function of DUSP2 leads not only to the prolonged activation of ERK and tumorigenesis, but also contributes to drug resistance. Low expression levels of DUSP5 and DUSP6 are involved in cetuximab resistance in head and neck squamous cell carcinoma (17). The present study examined whether DUSP2 is associated with cetuximab resistance in CRC. A plasmid carrying cDNA of DUSP2 was transfected into HCT15 and HCT116 cells. HCT15 and HCT116 cells harboring a KRAS mutation are primarily resistant to cetuximab. After $48 \mathrm{~h}$ of cetuximab treatment in the two cell lines, apoptosis was determined via flow cytometry using an Annexin V-FITC/PI staining kit. As shown in Fig. 5A, the overexpression of DUSP2 sensitized CRC cells to cetuximab, compared with the control plasmid $(\mathrm{P}<0.05)$. To investigate the function of DUSP2 on the sensitivity of CRC cell lines to cetuximab, the viability of HCT15 and HCT116 cells incubated in the presence of different cetuximab concentrations was evaluated using a cell viability assay (CCK-8 assay). As shown in Fig. 5B, the overexpression of DUSP2 enhanced the inhibitory effect of cetuximab on HCT15 and HCT116 cells, compared with the control plasmid. The present study also examined whether the inhibition of DUSP2 results in resistance to cetuximab in CRC cells. This analysis was performed in SW48 cells, which are sensitive to cetuximab. It was found that the knockdown of DUSP2 significantly inhibited the sensitivity of SW48 cells to cetuximab, compared with negative control (data not shown).

\section{Discussion}

CRC is the most common type of malignancy with the third highest incidence and mortality rates among all diagnosed cases of cancer worldwide $(18,19)$. Cetuximab combined with chemotherapy has demonstrated therapeutic efficacy in patients with metastatic CRC with all RAS wild-type tumors (20). However, the efficacy of cetuximab is limited by the development of resistance mechanisms in cancer cells (21). 

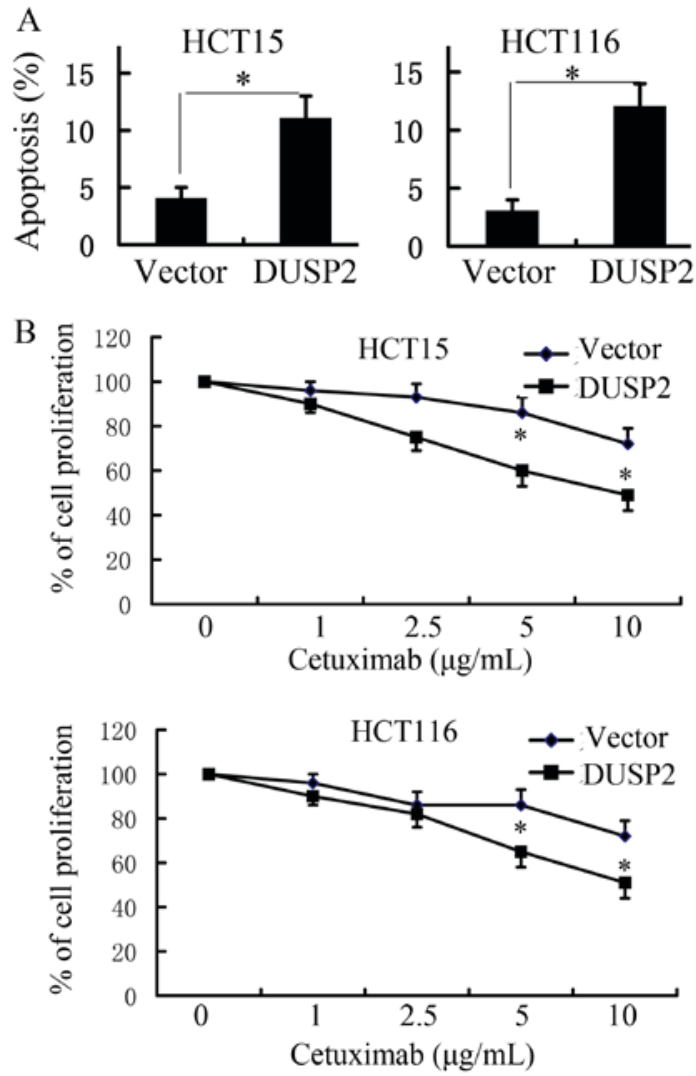

Figure 5. Enforced expression of DUSP2 enhances the sensitivity of HCT116 and HCT15 cells to cetuximab. (A) HCT116 and HCT15 cells stably transfected with plasmids carrying DUSP2 cDNA were treated with cetuximab at $20 \mu \mathrm{g} / \mathrm{ml}$ and the apoptotic cell populations were analyzed using flow cytometry. Data are presented as the mean \pm standard deviation $(n=3)$. ${ }^{*} \mathrm{P}<0.05$. (B) Cell viability was determined using a Cell Counting Kit-8 assay in the presence of different concentrations of cetuximab $(0,1,2.5,5$ and $10 \mu \mathrm{g} / \mathrm{ml})$. ${ }^{*} \mathrm{P}<0.05$. DUSP2, dual specificity phosphatase 2 .

Certain patients with all RAS wild-type tumors are primarily resistant to cetuximab (22). Increasing data has shown that the location of the primary tumor can be prognostic and predictive of responses to cetuximab in metastatic CRC, although the exact reason remains to be elucidated (23). The results of previous clinical studies have indicated that patients with left-sided RAS wild-type metastatic CRC require preferential treatment with cetuximab $(24,25)$. The latest National Comprehensive Cancer Network guideline for colon cancer recommends that cetuximab combination therapy is only used for left-sided RAS wild-type metastatic CRC (21). Significant differences have been observed to exist between LSCC and RSCC, with regard to epidemiological, biological and clinical data concerned with carcinogenesis and survival (26). Zhu et al reported that 11 genes, including DUSP2, were found to be differentially expressed in LSCC and RSCC by expression profiling with microarray analysis (27). In addition, the loss of DUSP2 promotes angiogenesis and metastasis via the upregulation of interleukin-8 in colon cancer (11).

In the present study, the expression of DUSP2 was investigated using IHC in 96 patients with CRC. It was found that the expression level of DUSP2 was significantly upregulated in CRC tissue, compared with that in paired normal colon tissue. The IHC analyses also demonstrated that the expression of DUSP2 in LSCC was significantly higher, compared with that in RSCC. Low expression levels of DUSP5 and DUSP6 are involved in cetuximab resistance in head and neck squamous cell carcinoma, and decreased expression of DUSP2 is associated with drug resistance in cells of several types of cancer. It has also been reported that the loss of function of DUSP2 leads to the prolonged activation of ERK (17). The EGFR-independent activation of the RAS/RAF/MAPK kinase/MAPK pathway is one of the resistance mechanisms to cetuximab (28). The present study examined whether DUSP2 is associated with cetuximab resistance in CRC. The results demonstrated that the overexpression of DUSP2 increased the inhibitory effect of cetuximab in CRC.

It is reported that the expression of DUSP2 is downregulated in several types of cancer in humans and that the loss of DUSP2 promotes cancer progression (2). By contrast, the present study found that the expression level of DUSP2 was significantly upregulated in CRC tissues, compared with that in paired normal colon tissues. It was hypothesized that the upregulation of DUSP2 may be a consequence rather than a cause of tumor progression in CRC cells. To address this question, the expression levels of DUSP2 and pERK were analyzed using IHC to determine whether the expression of DUSP2 was positively correlated with pERK in CRC tissues. It is known that EGF stimulation causes a significant increase in pERK and that this effect is observed even following EGF withdrawal. When the HCT15 and HCT116 cells were treated with hEGF to induce the expression of $\mathrm{pERK}$, it was found that the expression of DUSP2 was upregulated in HCT15 cells. It was also found that DAC and hEGF synergistically induced the expression of DUSP2, suggesting that the hypermethylation of DUSP2 may inhibit the upregulation of DUSP2 induced by the increase in pERK in HCT116 cells. The hypermethylation of DUSP2 was observed in 13/96 CRC tissues, and the primary tumors of these 13 patients all originated on the right side of the colon. When the expression levels of DUSP2 and pERK were analyzed in CRC tissues without hypermethylation of DUSP2, it was found that the expression of DUSP2 was positively correlated with pERK in CRC tissues. It was hypothesized that the upregulation of DUSP2 may function via negative feedback to balance the activation of MAPKs, including ERK1/2, p38 MAPK and $\mathrm{JNK}$, in CRC cells. This process may be inhibited by the hypermethylation of DUSP2 in RSCC.

In conclusion, the findings of the present study revealed that DUSP2 was overexpressed in LSCC and epigenetically silenced in RSCC. The overexpression of DUSP2 may be a consequence rather than a cause of tumor progression in CRC cells. The upregulation of DUSP2 may function via negative feedback to balance the activation of MAPKs, including ERK1/2, p38 MAPK and JNK, in CRC cells. Furthermore, the results demonstrated that the overexpression of DUSP2 increased the inhibitory effect of cetuximab in CRC, suggesting that DUSP2 may be a novel biomarker and therapeutic target in CRC therapy.

\section{Acknowledgements}

The present study was supported by the National Natural Science Foundation of China (grant no. 81672424) and the Youth Innovation Fund of the First Affiliated Hospital of Zhengzhou University (grant no. 20150101). 


\section{References}

1. Bermudez O, Pages G and Gimond C: The dual-specificity MAP kinase phosphatases: Critical roles in development and cancer. Am J Physiol Cell Physiol 299: C189-C202, 2010.

2. Wei W, Jiao Y, Postlethwaite A, Stuart JM, Wang Y, Sun D and Gu W: Dual-specificity phosphatases 2: Surprising positive effect at the molecular level and a potential biomarker of diseases. Genes Immun 14: 1-6, 2013.

3. Hamamura K, Nishimura A, Chen A, Takigawa S, Sudo A and Yokota H: Salubrinal acts as a Dusp2 inhibitor and suppresses inflammation in anti-collagen antibody-induced arthritis Cellular signalling 27: 828-835, 2015.

4. Perander M, Al-Mahdi R, Jensen TC, Nunn JA, Kildalsen H, Johansen B, Gabrielsen M, Keyse SM and Seternes OM: Regulation of atypical MAP kinases ERK3 and ERK4 by the phosphatase DUSP2. Sci Rep 7: 43471, 2017.

5. Yin Y, Liu YX, Jin YJ, Hall EJ and Barrett JC: PAC1 phosphatase is a transcription target of p53 in signalling apoptosis and growth suppression. Nature 422: 527-531, 2003.

6. Haag T, Richter AM, Schneider MB, Jimenez AP and Dammann RH: The dual specificity phosphatase 2 gene is hypermethylated in human cancer and regulated by epigenetic mechanisms. BMC Cancer 16: 49, 2016.

7. Lin SC, Chien CW, Lee JC, Yeh YC, Hsu KF, Lai YY, Lin SC and Tsai SJ: Suppression of dual-specificity phosphatase-2 by hypoxia increases chemoresistance and malignancy in human cancer cells. J Clin Invest 121: 1905-1916, 2011.

8. Lee YH, Morrison BL and Bottaro DP: Synergistic signaling of tumor cell invasiveness by hepatocyte growth factor and hypoxia. J Biol Chem 289: 20448-20461, 2014.

9. Karakashev SV and Reginato MJ: Hypoxia/HIF1 $\alpha$ induces lapatinib resistance in ERBB2-positive breast cancer cells via regulation of DUSP2. Oncotarget 6: 1967-1980, 2015.

10. Hou PC, Li YH, Lin SC, Lin SC, Lee JC, Lin BW, Liou JP, Chang JY, Kuo CC, Liu YM, et al: Hypoxia-Induced downregulation of DUSP-2 phosphatase drives colon cancer stemness. Cancer Res 77: 4305-4316, 2017.

11. Lin SC, Hsiao KY, Chang N, Hou PC and Tsai SJ: Loss of dual-specificity phosphatase-2 promotes angiogenesis and metastasis via up-regulation of interleukin-8 in colon cancer. J Pathol 241: 638-648, 2017.

12. Givant-Horwitz V, Davidson B, Goderstad JM, Nesland JM, Tropé CG and Reich R: The PAC-1 dual specificity phosphatase predicts poor outcome in serous ovarian carcinoma. Gynecol Oncol 93: 517-523, 2004

13. Zhu W, Huang L, Xu X, Qian H and Xu W: Anti-proliferation effect of BMI-1 in U937 cells with siRNA. Int J Mol Med 25: 889-895, 2010

14. Wang XF, Zhao YB, Wu Q, Sun ZH and Li HJ: Triptolide induces apoptosis in endometrial cancer via a p53 independent mitochondrial pathway. Mol Med Rep 9: 39-44, 2014.

15. Trastour C, Benizri E, Ettore F, Ramaioli A, Chamorey E, Pouysségur J and Berra E: HIF-lalpha and CA IX staining in invasive breast carcinomas: Prognosis and treatment outcome. Int J Cancer 120: 1451-1458, 2007.

16. Tashiro $\mathrm{E}$, Henmi $\mathrm{S}$, Odake $\mathrm{H}$, Ino $\mathrm{S}$ and Imoto $\mathrm{M}$ : Involvement of the MEK/ERK pathway in EGF-induced E-cadherin down-regulation. Biochem Biophys Res Commun 477: 801-806, 2016.
17. Boeckx C, Op de Beeck K, Wouters A, Deschoolmeester V, Limame R, Zwaenepoel K, Specenier P, Pauwels P, Vermorken JB, Peeters M, et al: Overcoming cetuximab resistance in HNSCC: The role of AURKB and DUSP proteins. Cancer Lett 354: 365-377, 2014.

18. Chen Y, Huang Y, Hou P, Zhang Z, Zhang Y, Wang W, Sun G, $\mathrm{Xu}$ L, Zhou J, Bai J and Zheng J: ING4 suppresses tumor angiogenesis and functions as a prognostic marker in human colorectal cancer. Oncotarget 7: 79017-79031, 2016.

19. Siegel RL, Miller KD and Jemal A: Cancer statistics, 2016. CA Cancer J Clin 66: 7-30, 2016.

20. Zhang X, Song Y, Song N, Zhang Y, Zhang L, Wang Y, Wang Z, Qu X and Liu Y: RANKL/RANK pathway abrogates cetuximab sensitivity in gastric cancer cells via activation of EGFR and c-Src. Onco Targets Ther 10: 73-83, 2017.

21. Taieb J, Balogoun R, Le Malicot K, Tabernero J, Mini E, Folprecht G, Van Laethem JL, Emile JF, Mulot C, Fratté S, et al: Adjuvant FOLFOX +/-cetuximab in full RAS and BRAF wildtype stage III colon cancer patients. Ann Oncol 28: 824-830, 2017.

22. Queralt B, Cuyàs E, Bosch-Barrera J, Massaguer A, de Llorens R, Martin-Castillo B, Brunet J, Salazar R and Menendez JA: Synthetic lethal interaction of cetuximab with MEK1/2 inhibition in NRAS-mutant metastatic colorectal cancer. Oncotarget 7: 82185-82199, 2016.

23. Wang F, Bai L, Liu TS, Yu YY, He MM, Liu KY, Luo HY, Zhang DS, Jin Y, Wang FH, et al: Right-sided colon cancer and left-sided colorectal cancers respond differently to cetuximab. Chin J Cancer 34: 384-393, 2015.

24. Brule SY, Jonker DJ, Karapetis CS, O'Callaghan CJ, Moore MJ, Wong R, Tebbutt NC, Underhill C, Yip D, Zalcberg JR, et al: Location of colon cancer (right-sided versus left-sided) as a prognostic factor and a predictor of benefit from cetuximab in NCIC CO.17. Eur J Cancer 51: 1405-1414, 2015.

25. Moretto R, Cremolini C, Rossini D, Pietrantonio F, Battaglin F, Mennitto A,BergamoF,LoupakisF,MarmorinoF,Berenato R, et al: Location of primary tumor and benefit from anti-epidermal growth factor receptor monoclonal antibodies in patients with RAS and BRAF wild-type metastatic colorectal cancer. Oncologist 21: 988-994, 2016.

26. Dienstmann R, Vermeulen L, Guinney J, Kopetz S, Tejpar S and Tabernero J: Consensus molecular subtypes and the evolution of precision medicine in colorectal cancer. Nat Rev 17: 79-92, 2017.

27. Zhu H, Wu TC, Chen WQ, Zhou LJ, Wu Y, Zeng L and Pei HP: Screening for differentially expressed genes between left- and right-sided colon carcinoma by microarray analysis. Oncol Lett 6: 353-358, 2013

28. Joo D, Woo JS, Cho KH, Han SH, Min TS, Yang DC and Yun $\mathrm{CH}$ : Biphasic activation of extracellular signal-regulated kinase (ERK)1/2 in epidermal growth factor (EGF)-stimulated SW480 colorectal cancer cells. BMB Rep 49: 220-225, 2016

(i) (9) This work is licensed under a Creative Commons Attribution-NonCommercial-NoDerivatives 4.0 International (CC BY-NC-ND 4.0) License. 